\title{
DIFFRACTION AND TRAPPING OF WAVES BY \\ CAVITIES AND SLENDER BODIES
}

\author{
GRANT R. Bigg
}

This thesis consists of two parts, linked by the use of common mathematical techniques and the consideration of similar physical phenomena. Part I examines the response of cavities to external exciting oscillations, in both two and three dimensions. The cavities are assumed to possess openings whose dimensions are small compared to the size of the cavity itself. This assumption enables the technique of matched asymptotic expansions to be employed. For a portion of the analysis the further assumption that the wavelength of the exciting oscillation is large is used. This enables a careful inspection to be made of the Helmholtz resonance of the cavity.

The theory is compared to numerical computations, made using a boundary integral equation technique, and work, both analytic and experimental, by other researchers in this field. Several interesting results of the theory are discussed, such as the symmetry property which enables the number of cavities treatable by this analysis to be extended and the difference in response curve for cavities of the same volume.

The second part of the thesis examines the scattering of waves by slender bodies which are either transparent to the incident radiation or opaque to it. The specific application of the work is to an oceanographic situation but simple extensions may be made to include acoustic, electromagnetic and elastic problems. The work of Mei and Tuck [1], which employed the parabolic approximation to study scattering by (mostly)

Received 7 April 1983. Thesis submitted to University of Adelaide, November 1982. Degree approved March 1983. Supervisor: Professor E.0. Tuck. 
transparent bodies, is analysed critically using matched asymptotic expansions and numerical comparisons; these using boundary integral equation methods. The existence of a special class of "barely submerged islands" is verified but it is shown that the theory is not useful when energy is trapped over the feature, producing resonance.

Part II also examines, both numerically and analytically, the scattering of waves by slender opaque bodies, or "islands". Matched asymptotic expansion analysis reveals that the parabolic approximation, which essentially says that the amplitude of the wave varies more rapidly in one direction than another, is useful over a range of incident wavelengths but not uniformly.

\section{Reference}

[1] C.C. Mei and E.O. Tuck, "Forward scattering by long thin bodies", SIAM J. App Z. Math. 39 (1980), 178-191.

Department of Applied Mathematics, University of Adelaide, GPO Box 498, Adelaide, South Australia 5001 , Australia. 